\title{
Cell fate patterning during $C$. elegans vulval development
}

\author{
Russell J. Hill and Paul W. Sternberg \\ Howard Hughes Medical Institute, Division of Biology 156-29, California Institute of Technology, Pasadena CA 91125, USA
}

\section{SUMMARY}

Precursor cells of the vulva of the $C$. elegans hermaphrodite choose between two vulval cell fates $\left(1^{\circ}\right.$ and $\left.2^{\circ}\right)$ and a non-vulval epidermal fate $\left(3^{\circ}\right)$ in response to three intercellular signals. An inductive signal produced by the anchor cell induces the vulval precursors to assume the $1^{\circ}$ and $2^{\circ}$ vulval fates. This inductive signal is an EGFlike growth factor encoded by the gene lin-3. An inhibitory signal mediated by lin-15, and which may originate from the surrounding epidermis, prevents the vulval precursors from assuming vulval fates in the absence of the inductive signal. A short range lateral signal, which acts through the gene $\operatorname{lin}-12$, regulates the pattern of 1 and 2 fates assumed by the induced vulval precursors.
The combined action of the three signals precisely directs the six vulval precursors to adopt a $3^{\circ} 3^{\circ} 2^{\circ} 1^{\circ} 2^{\circ}$ 3 pattern of fates. The amount of inductive signal produced by the anchor cell appears to determine the number of vulval precursors that assume vulval fates. The three induced vulval precursors most proximal to the anchor cell are proposed to adopt the $2^{\circ} 1^{\circ} 2^{\circ}$ pattern of fates in response to a gradient of the inductive signal and also in response to lateral signalling that inhibits adjacent vulval precursor cells from both assuming the $1{ }^{\circ}$ fate.

Key words: development, genetics, C. elegans, vulva, EGF, intercellular signalling

\section{INTRODUCTION}

The nematode Caenorhabditis elegans has been used to study the mechanisms that control cell fate determination (reviewed by Greenwald and Rubin, 1992; Lambie and Kimble, 1991a). The development of C. elegans follows a largely invariant cell lineage (Kimble and Hirsh, 1979; Sulston and Horvitz, 1977; Sulston, 1976; Sulston et al., 1980; Sulston et al., 1983). Although some of the cell lineages of $C$. elegans appear to be specified by cellautonomous mechanisms, other lineages are specified by cell-cell interactions. Some of these interactions were first noted from instances of naturally occurring variation in the lineage in which the development of a cell correlated with its position, thus suggesting that the cell was responding to environmental cues (Sulston and Horvitz, 1977). Other examples of cell-cell interactions have been defined by experiments in which groups of cells are ablated by irradiation with a laser microbeam to see if the development of the remaining cells is affected by the change in their environment (Kimble, 1981; Priess and Thomson, 1987; Schnabel, 1991: Sulston et al., 1983; Sulston and White, 1980). C. elegans is well suited for genetic analysis (Brenner, 1974) and many mutations that affect its cell lineage have been identified (Horvitz, 1988). The genetic analysis of these mutations and the characterization of the corresponding genes with the techniques of molecular biology has been a driving force in elucidating the mechanisms that control cell fate in C. elegans.
The development of the hermaphrodite vulva has been extensively studied by cell ablation experiments and by genetic analysis. The results of these studies support a three signal model of vulval development summarized below (and reviewed by Horvitz and Sternberg, 1991) (Fig. 1). The vulva normally develops from three of six ectodermal blast cells called Vulval Precursor Cells (VPCs) that are located within the ventral epidermis. Although in wild-type development each of the VPCs always assumes a particular fate, a variety of experiments indicate that each of the VPCs is equivalent in its ability to assume either of three fates and thus the VPCs constitute an equivalence group (Sternberg and Horvitz, 1986; Sulston and White, 1980; Thomas et al., 1990). Each fate consists of a distinct cell lineage that produces a particular set of cell types (Fig. 2). The $1^{\circ}$ and $2^{\circ}$ fates both produce vulval tissue although they contribute to different regions of the vulva. The $3^{\circ}$ fate produces nonvulval epidermis (Sulston and Horvitz, 1977; Sulston, 1976). The fate chosen by a VPC depends upon three intercellular signals. The anchor cell of the somatic gonad produces a signal that induces the three more proximal VPCs to assume the $1^{\circ}$ and $2^{\circ}$ fates (Kimble, 1981). An inhibitory signal inhibits the VPCs from assuming vulval fates in the absence of the inductive signal (Herman and Hedgecock, 1990). A lateral signal among the induced VPCs regulates the pattern of $1^{\circ}$ and $2^{\circ}$ fates (Sternberg, 1988a). In wild-type development the combined action of the three signals causes the six VPCs to assume a $3^{\circ} 3^{\circ} 2^{\circ} 1^{\circ} 2^{\circ} 3^{\circ}$ pattern of fates. In the following sections, each of the three 


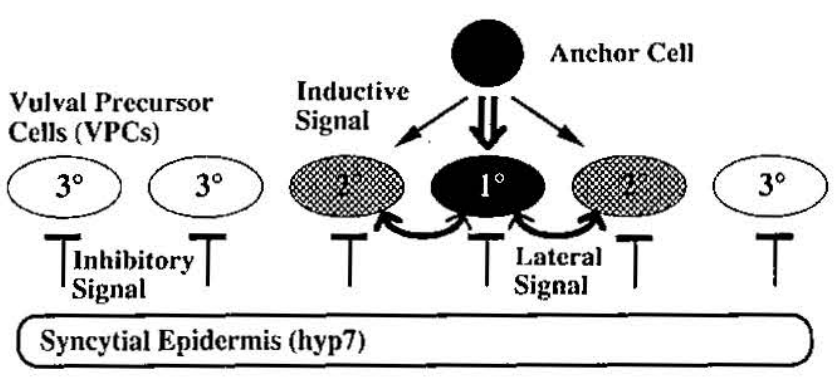

Fig. 1. Three-signal model of VPC fate determination. The six VPCs choose between three possible fates, $1^{\circ}, 2^{\circ}$ and $3^{\circ}$ in response to three intercellular signals. A spatially graded inductive signal made by the anchor cell induces the three more proximal VPCs to assume $1^{\circ}$ and $2^{\circ}$ vulval fates. An inhibitory signal from the syncytial epidermis prevents the VPCs from assuming vulval fates in the absence of the inductive signal. A lateral signal acts among the VPCs to promote the $2^{\circ}$ fate. Each VPC is believed to laterally signal its neighbors in proportion to the amount of inductive signal that it receives.

signals is discussed separately. This is then followed by a discussion of how the pattern of VPC fates is controlled by the three signals.

\section{THE INDUCTIVE PATHWAY}

Cell ablation experiments indicate that the vulva develops as the result of an inductive interaction between the somatic gonad and the vulval precursors (Sulston and White, 1980). Kimble showed that the anchor cell of the somatic gonad is necessary and sufficient to induce vulva development (Kimble, 1981). If the anchor cell or its precursors are ablated, then the three VPCs that would normally assume the $1^{\circ}$ and $2^{\circ}$ fates instead assume the $3^{\circ}$ fate (Table $1 \mathrm{C}$ ). This induction occurs 1-3 hours before the VPCs divide (Kimble, 1981). If the anchor cell is ablated after the VPCs have divided, the cell lineage of their daughters is unaffected (Kimble, 1981; Sternberg and Horvitz, 1986). A large number of mutations that disrupt vulva induction have been characterized (reviewed by Horvitz and Sternberg, 1991). The cloning of several of these genes indicates that this induction is mediated by an Epidermal Growth Factor (EGF)-like signalling pathway that includes an EGF-like growth factor encoded by lin-3 (Hill and Sternberg, 1992) and an EGF-Receptor like molecule encoded by let-23 (Aroian et al., 1990).

\section{lin-3 encodes the inductive signal}

Lin-3 is proposed to be a nematode member of the Epidermal Growth Factor (EGF) family of growth factors (Hill and Sternberg, 1992). This family includes ligands of the EGF-Receptor (EGF-R) such as EGF (Gregory, 1975; Savage et al., 1972). Transforming Growth Factor-alpha (TGF- $\alpha$ ) (Derynck et al., 1984), Heparin Binding-EGF (HBEGF) (Higashiyama et al., 1991), amphiregulin (Shoyab et al., 1989), and also ligands of neu/HER2, a homologue of the EGF-R, (Holmes et al., 1992, Wen et al., 1992) and the predicted product of the spitz locus of Drosophila (Rutledge et al., 1992). The EGF growth factors are usually made as

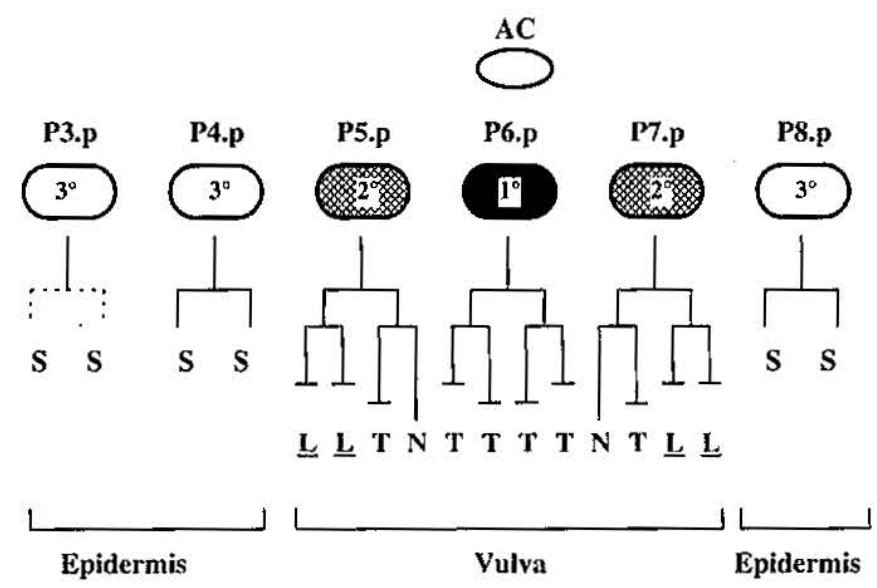

Fig. 2. Schematic representation of the fates of the VPCs in wildtype vulval development. The name, relative position, and fate of each cell is shown. The lineage associated with each fate is drawn below the VPCs. Vertical lines represent cells and horizontal lines represent cell divisions. P5.p, P6.p, and P7.p contribute to the vulva and the other VPCs form epidermis. P3.p can assume the $3^{\circ}$ fate or fuse directly to the syncytial epidermis without dividing (Sulston and Horvitz, 1977, Sulston and White, 1980). A unique characteristic associated with the final division of each cell, or with the terminal progeny, is indicated by a single letter code beneath the lineage, (convention of Sternberg and Horvilz, 1986): $S$, cell fuses with the syncytial epidermis hyp $7 ; \mathrm{L}$, nucleus divides longitudinally in the anterior-posterior axis of the body, underline indicates that the daughter nuclei adhere to the ventral cuticle; T, nucleus divides transversely in the left-right body axis; $\mathrm{N}$, nucleus does not divide. AC: Anchor Cell.

membrane spanning proteins that contain at least one extracellular EGF domain (reviewed by Carpenter and Wahl, 1990). EGF domains are sequence motifs of approximately 50 amino acids that consist of six cysteine residues with semi-conserved spacing (reviewed by Davis, 1990). In many cases it is known that the EGF domain can be processed away from the rest of the protein to produce a secreted factor that can activate its receptor (reviewed by Carpenter and Wahl, 1990). In the case of TGF- $\alpha$, the EGF domain can also activate the receptor without being released from the membrane (reviewed by Massagué, 1990).

The gene lin-3 was identified by reduction-of-function mutations that cause a recessive, vulvaless phenotype in which up to all three of the VPCs that usually assume vulval fates instead assume epidermal fates (Ferguson and Horvitz, 1985; Horvitz and Sulston, 1980; Sulston and Horvitz, 1981). Thus, wild-type $\operatorname{lin}-3$ activity is required for vulval induction. In contrast, animals bearing lin-3 transgenes (transgenes made up of wild-type genomic DNA cloned from the lin-3 locus) can have a dominant multivulva phenotype in which up to all six of the VPCs assume vulval fates (Hill and Sternberg, 1992). The multivulva phenotype of the transgenes is proposed to be a gain-of-function phenotype because the transgenes are created by a method that concatenates hundreds of copies of the injected DNA into an extrachromosomal array (Mello et al., 1991). Such an array would likely express levels of Lin-3 protein that are greater than the levels expressed by the two chromosomal copies of the lin-3 locus. Together, the reduction-of-function 
Table 1. Vulval Fate Patterns

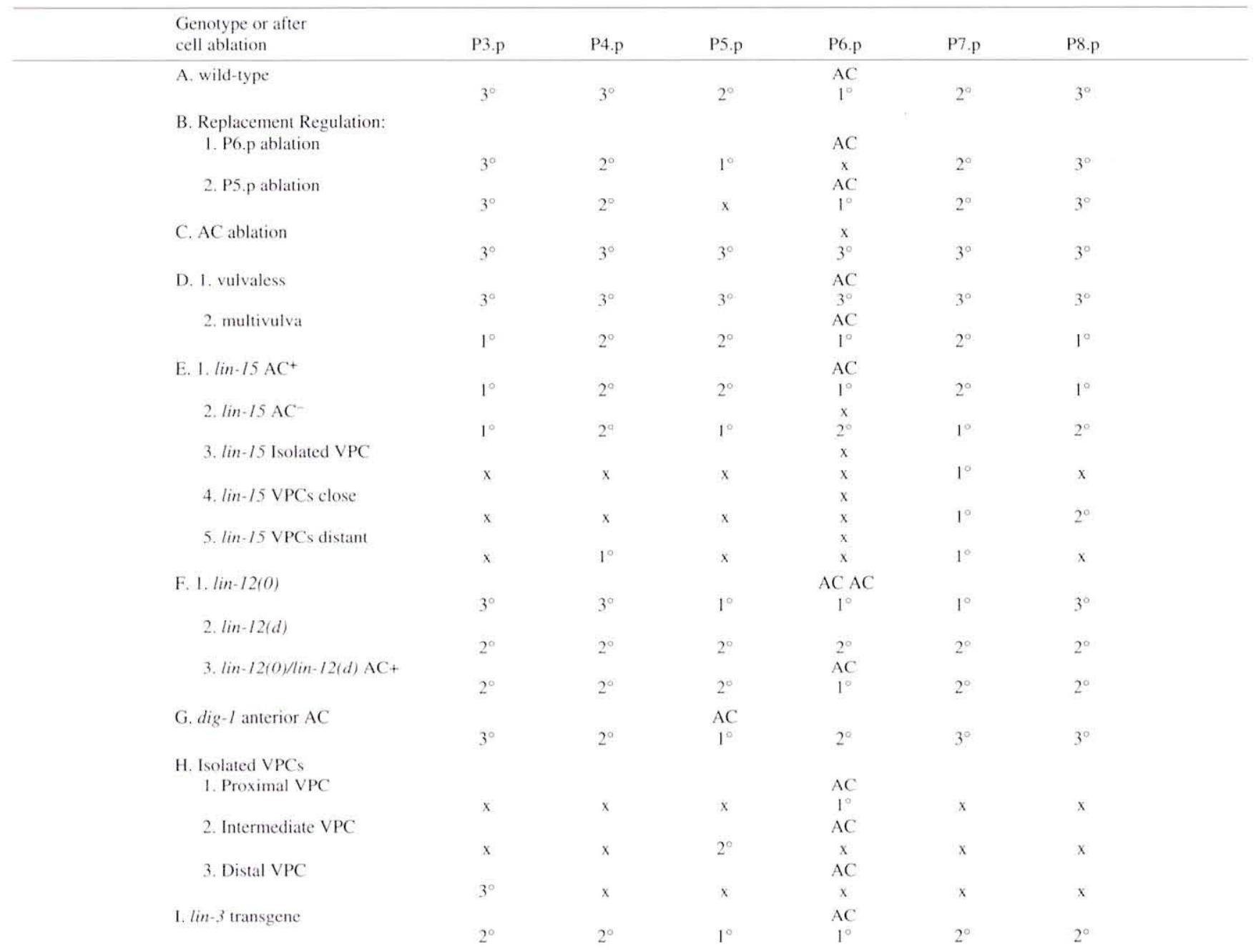

(A) In wild-type development the six VPCs assume the pattern of fates $3^{\circ} 3^{\circ} 2^{\circ} 1^{\circ} 2^{\circ} 3^{\circ}$ (Sulston and Horvitz, 1977. Sulston. 1976, Sulston and White, 1980). (B) The VPCs can display regulation of fates upon ablation of VPCs prior to the time of vulval induction. A cell that normally adopts the $3^{\circ}$ or $2^{\circ}$ fate can assume the $1^{\circ}$ fate if the cell that normally assumes the $1^{\circ}$ fate is destroyed. A cell that normally assumes the $3^{\circ}$ fate can replace a $2^{\circ}$ cell. A $1^{\circ}$ cell will not replace a $2^{\circ}$ or $3^{\circ}$ cell, and a $2^{\circ}$ cell will not replace a $3^{\circ}$ cell. The regulating cell will usually assume the position, as well as the fate, of the cell that it is replacing. The abilitity of the VPCs to undergo replacement regulation supports the model that the VPCs are equivalent in their potential to assume either of the three fates (Sulston and White, 1980). (C) Ablation of the AC or its precursors before or during the first several hours of the L3 stage prevents P5.p. P6.p and P7.p from assuming vulval fates. Therefore, the vulval fates are specified by an inductive signal from the AC of the somatic gonad (Kimble, 1981; Sulston and White, 1980). (DI) Recessive mutations in the genes $\operatorname{lin}-2, \operatorname{lin}-3, \lim -7, \operatorname{lin}-10, \operatorname{lin}-45, \operatorname{let}-23, \operatorname{let}-60$, and $\operatorname{sem}-5$, and dominant negative mutations in let-60 can cause a vulvaless phenotype in which fewer than normal VPCs assume vulval fates, even though the AC and the VPCs are present (Aroian and Sternberg, 1991; Beitel et al., 1990; Clark et al.. 1992; Ferguson and Horvitz, 1985; Han et al. 1990, 1993; Horvitz and Sulston, 1980; Sulston and Horvitz, 1981). These genes are involved either in the generation of the inductive signal by the AC, or the response to the inductive signal by the VPCs. (D2) Recessive mutations in lin-I, and lin-15, and gain-of-function mutations of let-60, confer a multivulva phenotype in which greater than the normal number of VPCs assume vulval fates (Beitel et al., 1990; Ferguson and Horvitz, 1985; Han et al., 1990; Horvitz and Sulston, 1980: Sulston and Horvitz, 1981). (E) In lin-15 mutant animals all six VPCs assume vulval fates (E1) and can do so independently of the inductive signal (E2) (Ferguson and Horvitz, 1985; Ferguson et al., 1987). In lin-15 mutant animals it is commonly observed that two adjacent VPCs both assume the $2^{*}$ fate, but it is rarely observed that two adjacent VPCs both assume the $1^{\circ}$ fate. This suggests the action of a lateral signal among the VPCs that prevents two adjacent VPCs from both assuming the $1^{\circ}$ fate, (E1) In lin-15 mutant animals, the patterning of P5.p. P6.p. and P7.p is normal if the AC is present. P6.p always assumes the $1^{\circ}$ fate and P5.p and P7.p assume the $2^{\circ}$ fate. (E2) If the AC is absent, then the patterning of P5.p. P6.p and P7.p is disrupted such that any of these cells can assume either the $1^{\circ}$ or $2^{\circ}$ fate. Thus, the VPCs can still respond to the inductive signal in lin- 15 mutant animals. (E3) An isolated VPC in a lin- 15 mutant assumes the $1^{\circ}$ fate. (E4,5) Two isolated VPCs in a lin-15 mutant animal will usually both assume the $1^{\circ}$ fate if they are not in close proximity to each other, but only one of the cells will assume the $1^{\circ}$ fate if they remain in close contact with each other. Thus, the lateral signal between the VPCs appears to act only at a close range and may require direct cell contact (Sternberg. 1988a). (F) lin-12 activity promotes the $2^{\circ}$ fate in the VPC equivalence group and in the AC $\left(1^{\circ}\right)$ VU $\left(2^{\circ}\right)$ equivalence group (Greenwald et al., 1983). (F1) In animals homozygous for null mutations of $l i n-12$ approximately three VPCs are induced to assume $1^{\circ}$ lineages and there are often two or more ACs. (F2) In animals with dominant gain-of-function mutations in lin-12, all six VPCs assume $2^{\circ}$ fates and there is often no AC. (F3) In some animals of the genotype lin-12(d)/lin-12(0) $\mathrm{ACs}$ are present. When an $\mathrm{AC}$ is present. P6.p assumes the $1^{\circ}$ fate and when the $\mathrm{AC}$ is absent P6.p assumes the $2^{\circ}$ fate. Therefore the inductive signal can override the action of $l i n-12(d)$ mutations to specify the $1^{\circ}$ fate (Sternberg and Horvitz, 1989). (G) The AC is the organizing center of vulval fate patterning. When the AC is shifted to a more anterior position in dig-I mutant animals, the pattern of vulval development is usually commensurably shifted (Thomas et al., 1990). (H) The fate of an isolated VPC correlates with its distance from the AC. A VPC that is close to the AC will assume the ${ }^{\circ}$ fate, a VPC that is relatively further away will assume the $2^{\circ}$ fate, and a VPC that is far from the AC will assume the $3^{\circ}$ fate. This suggests that the inductive signal is distributed in a spatial gradient centered on the AC and that the VPCs can assume different fates in response to different levels of the inductive signal (Sternberg and Horvitz, 1986). (I) In animals bearing multicopy lin-3 transgenes, which presumably overexpress Lin-3 protein from the anchor cell, adjacent VPCs often assume the $1^{\circ}$ fate. Therefore, high levels of the inductive signal can override the effect of the lateral signal and specify two adjacent cells to both have the $1^{\circ}$ fate (Hill and Sternberg, unpublished data), AC: Anchor Cell. 


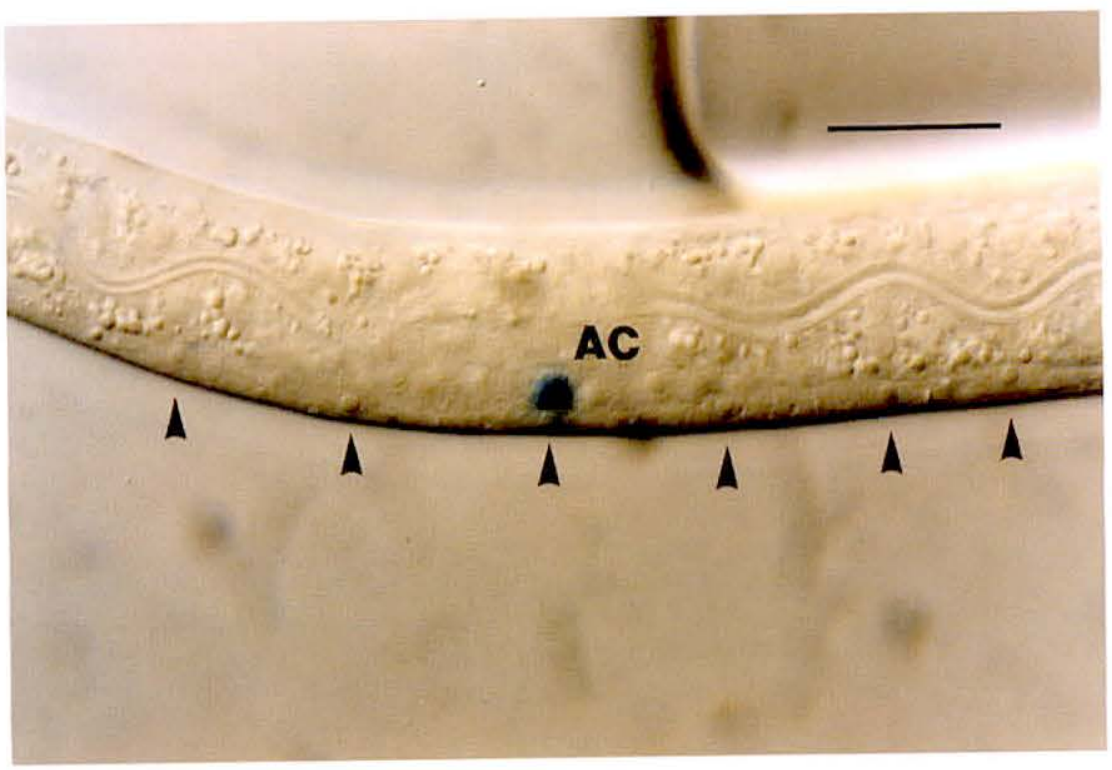

Fig. 3. A transgene that contains the lac $Z$ gene of $E$. coli inserted within a lin-3 genomic clone expresses a Lin-3:: $\beta$-gal fusion protein specifically in the anchor cell at the time of vulval induction. $\beta$-galactosidase activity is detected by histochemical staining with $\mathrm{X}$-gal (blue). Arrowheads show the positions of the nuclei of the six VPCs (P[3-8].p). AC: anchor cell. Scale bar equal to $20 \mu \mathrm{m}$. Adapted and reprinted with permission from Nature (358, 470-476) Copyright (1992) Macmillan Magazines Limited. (Hill and Sternberg, 1992.) and the gain-of-function phenotypes of lin-3 suggest that the dose of lin-3 activity controls the number of VPCs that assume vulval fates.

The analysis of lin-3 transgenes suggests that lin-3 can act in the anchor cell to cause the VPCs to assume vulval fates (Hill and Sternberg, 1992). A transgene in which the lacZ gene of $E$. coli is inserted in frame within a lin-3 genomic DNA clone directs expression of $\beta$-galactosidase activity specifically in the anchor cell at the time of vulval induction (Fig. 3). This transgene, which should produce a fusion protein that contains the extracellular and transmembrane domains of the Lin-3 protein and a cytoplasmic domain consisting of the $\beta$-galactosidase protein, retains the ability to induce vulval fates. This result suggests that expression of lin-3 in the anchor cell is sufficient to induce vulval fates. This hypothesis is further substantiated by the results of ablation experiments. Ablation of the four gonadal precursor cells at hatching, which prevents the development of the anchor cell, greatly reduces the ability of lin-3 transgenes to induce vulval development.

It has been proposed that the inductive signal is a secreted factor because induction of vulval fates can occur without apparent direct contact between the anchor cell and the induced VPCs (Sternberg and Horvitz, 1986; Sulston and White, 1980; Thomas et al., 1990). As mentioned previously, the EGF growth factors can produce secreted factors that consist of an EGF domain. We have made a transgene to test whether a secreted form of the EGF domain of Lin3 , without the rest of the Lin-3 protein, would be sufficient to induce vulval fates (Hill and Sternberg, unpublished data) (Fig. 4). This transgene uses a heat shock promoter that should express the Lin-3 EGF domain in a tissue general manner (Stringham et al., 1992). The transgene is able to stimulate $1^{\circ}$ and $2^{\circ}$ vulval fates even when the gonadal precursors have been ablated prior to the development of the anchor cell. This result suggests that Lin-3 can act as a secreted factor in a manner similar to the EGF growth factors. Since overexpression of the EGF domain of Lin-3 is sufficient to induce vulval fates in the absence of the

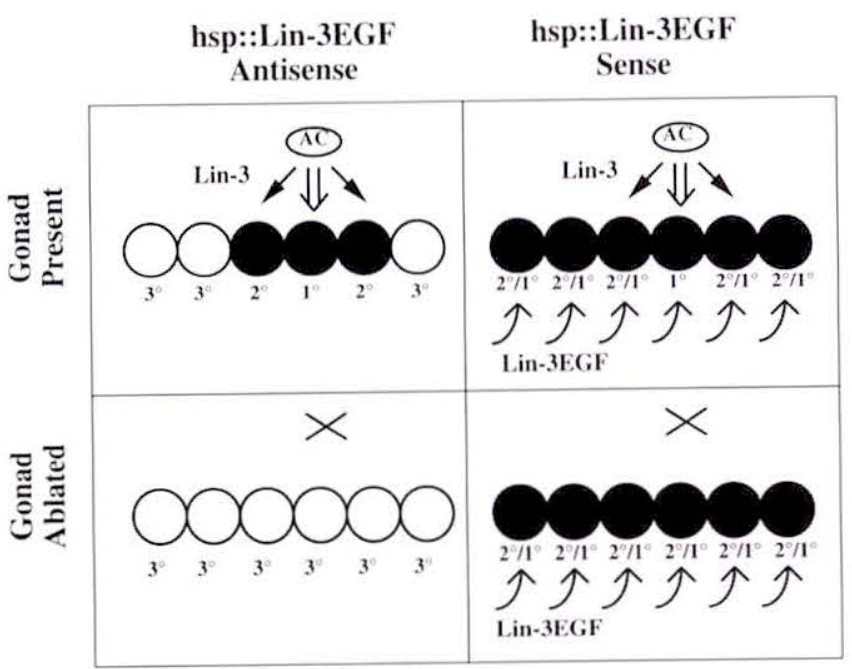

Fig. 4. The EGF domain of the Lin-3 protein can stimulate $1^{\circ}$ and $2^{\circ}$ vulval fates in the absence of the gonad. A transgene (sense) was designed that will secrete the EGF domain of the Lin-3 protein (Lin-3EGF) under the control of a tissue general heat shock promoter (Stringham et al., 1992). A control transgene (antisense) was designed that has the lin-3 DNA cloned in the antisense orientation to the promoter and which should produce no functional protein. These transgenes were placed in animals that are wild-type at the chromosomal lin-3 locus. Upon heat shock, animals bearing the antisense transgene have wild-type levels of vulval induction when the gonad is present and no vulval induction when the gonadal precursors are ablated at hatching. These observations demonstrate that the heat shock conditions do not affect the process of vulval induction and that the ablation conditions used completely remove the activity of the inductive signal made by the anchor cell. Upon heat shock, animals bearing the sense transgene display excess levels of vulval induction when the gonad is present and also when the gonadal precursors have been ablated. (Adapted from Hill and Sternberg, unpublished data.)

gonad, it is possible that Lin-3 is the only vulval inducing signal made specifically by the anchor cell. It is not yet known if the Lin-3 protein is normally processed in vivo. 


\section{The response pathway of the VPCs}

A pathway of genes has been characterized that is believed to mediate the response of the VPCs to the inductive and inhibitory signals (Fig. 5). This pathway includes the following genes, which are believed to act in the following order on the basis of genetic epistasis experiments: let-23, which encodes a homologue of the EGF-Receptor (EGF-R) (Aroian et al., 1990); sem-5, which encodes an adapter protein that contains SH2 and SH3 domains (Clark et al., 1992); let-60, which encodes a ras protein (Han and Sternberg, 1990); and lin-45, which encodes a raf serinethreonine kinase (Han et al., 1993). Wild-type activity of each of these genes is required for vulval induction. A reduction of function mutation in let-23 is epistatic to the ability of lin-3 transgenes to stimulate vulval fates indicating that let-23 acts downstream of lin-3. (Hill and Sternberg, 1992). This pathway is similar to pathways studied in other experimental systems. For example, a ras protein, a raf kinase, and a Sem-5-like adapter protein also act downstream of the receptors encoded by the torso (N. Perrimon. this Volume) and sevenless (E. Hafen, this Volume) genes of Drosophila, and also downstream of growth factor receptors in mammals (McCormick, 1993).

A number of other genes have been implicated in the process of vulval induction. The genes lin-2, lin-7, and lin-10 are required for wild-type levels of vulval induction (Ferguson and Horvitz, 1985). These genes may act at a step near let-23 in the pathway of vulval induction (Ferguson et al., 1987). The gene lin-10 encodes a novel protein (Kim and Horvitz, 1990). The gene lin-1 acts downstream of lin-45(Han et al., 1993). Wild-type lin- $I$ activity acts to repress vulval fates. (Ferguson and Horvitz, 1985; Ferguson et al., 1987).

lin-3 and let-23 are both required for vulval induction. larval viability (Aroian and Sternberg, 1991; Clark et al., 1988; Ferguson and Horvitz, 1985; Herman, 1978, and R. Hill and P. Sternberg, unpublished results), hermaphrodite fertility (Aroian and Sternberg, 1991; Ferguson and Horvitz, 1985 ) and proper specification of cell fate in the male B lineage $(H$. Chamberlin and P. Sternberg, unpublished results). This suggests that the same growth factor and receptor are used together in multiple decisions during the development of $C$. elegans. let-23 is also required for proper cell fate specification in the P11-P12 equivalence group (Aroian and Sternberg, 1991), but it has not been demonstrated whether lin-3 is also required for P11-P12 fate specification. sem-5 (Clark et al., 1992), let-60 (Beitel et al., 1990; Clark et al., 1988; Han et al., 1990), and lin-45 (Han et al., 1993) are also required for larval viability and a simple model is that they act downstream of let-23 in all tissues that use the lin-3 let-23 pathway. lin-2, lin-7, and lin-10, are unique in that they are only required for vulval induction (Ferguson and Horvitz, 1985). In contrast, sem-5 may be a generalized signal transduction component that acts downstream of multiple receptors, since sem-5 activity is required for the proper positioning of the sex myoblasts (Clark et al., 1992), a process that does not require lin-3 or let-23.

\section{THE INHIBITORY PATHWAY}

Genetic experiments suggest that a pathway of genes that

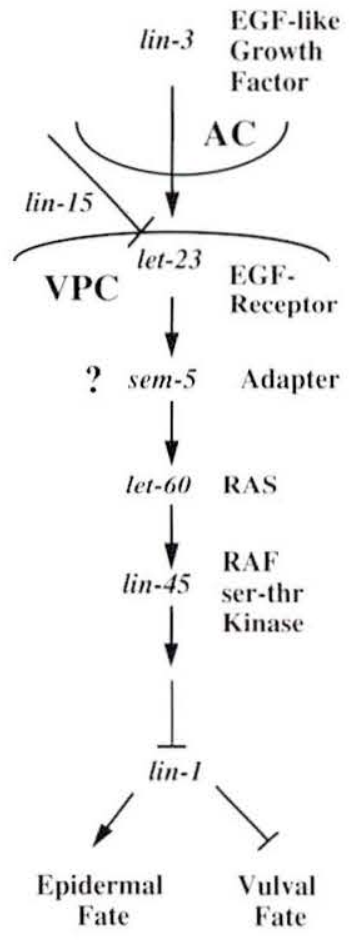

Fig. 5. Simplified pathway of vulval induction. The gene lin-3 encodes a EGF-like growth factor made by the anchor cell that is proposed to activate let 23 . lin- 15 negatively regulates the basal activity of let-23. let-23 encodes an EGF-Receptor-like tyrosine kinase. An adapter protein encoded by sem-5 is proposed to couple the action of let-23 to let-60. let-60 encodes a ras protein that acts upstream of the raf ser-thr kinase encoded by lin-45. Activity of this pathway eventually results in inactivation of lin-l. lin-l promotes the epidermal fate and inhibits the vulval fate.

includes lin-15 (Ferguson and Horvitz, 1985; Ferguson and Horvitz, 1989) produces an intercellular signal that inhibits vulval fates (Herman and Hedgecock, 1990). Loss-offunction mutations in the lin-15 locus cause a multivulva phenotype in which all six VPCs can assume vulval fates (Table IE1). lin-15 mutant hermaphrodites display a multivulva phenotype even if the anchor cell has been ablated (Table 1E2). Thus, lin-15 does not act only in the anchor cell (Ferguson et al., 1987). Genetic mosaic experiments indicate that lin-15 does not act cell autonomously in the VPCs, and therefore that lin-15 is part of an intercellular signalling pathway (Herman and Hedgecock, 1990). The overall pattern of mosaic phenotypes is complex and has been interpreted to indicate that lin-15 can act in the epidermal syncytium hyp7, a tissue generated by several different cell lineages (Herman and Hedgecock, 1990). hyp7 is the main body epidermis and surrounds all of the VPCs. Thus a simple model is that the inhibitory signal would affect all of the VPCs equivalently and would thus provide no spatial specificity.

The inhibitory signal appears to act in parallel to the inductive signal to regulate the activity of the response pathway of the VPCs. Reduction-of-function mutations in the let-23 locus that confer a vulvaless phenotype are epistatic to the multivulva phenotype of lin-15 mutations (Aroian and Sternberg, 1991; Ferguson et al., 1987; L. Huang and P. Sternberg, unpublished data). Therefore, lin- 
15, like the inductive signal, is an upstream regulator of let23. The inductive and inhibitory signals can regulate vulval fates independently of each other suggesting that they act in parallel. First, in a mutant animal that lacks lin-15 activity, the cell P6.p will always assume the $1^{\circ}$ fate if the anchor cell is present, but can assume the $2^{\circ}$ or $1^{\circ}$ fate if the anchor cell is absent (Table 1E1,2). This indicates that the inductive signal can influence vulval fate choice independently of $/ \mathrm{in}$ 15 (Sternberg, 1988a). Second, the multivulva phenotype of putative null mutations of $\mathrm{lin}-15$ are epistatic to the vulvaless phenotype of strong reduction-of-function $\mathrm{lin}-3$ genotypes. (Ferguson el al., 1987; R. Hill and P. Sternberg unpublished results). This suggests that the inhibitory signal can affect vulval fates independently of the inductive signal encoded by lin-3. The inhibitory signal does not appear to be a competitive inhibitor of the inductive signal since multicopy lin-15 transgenes that presumably overexpress the Lin-15 protein do not interfere with vulval induction (L. Huang. and P. Sternberg, unpublished results). In wild-type development, a VPC that receives both the inductive signal and the inhibitory signal will assume a vulval fate. Thus, the inhibitory signal prevents the VPCs from assuming vulval fates only in the absence of the inductive signal. One possible interpretation of these observations is that the inhibitory signal negatively regulates the basal activity of let-23. The molecular mechanism by which this inhibitory pathway acts is not understood.

\section{THE LATERAL SIGNAL}

In addition to the inductive and inhibitory signals that originate from tissues other than the VPCs, a lateral signal among the VPCs also regulates VPC fate choice. Evidence for this signal came from observations on the pattern of VPC fates found in $\mathrm{lin}-15$ mutant animais (Sternberg, 1988a). As described above, all six VPCs assume $1^{\circ}$ and $2^{\circ}$ vulval fates in a lin-15 mutant animal due to a defect in an inhibitory signal from the surrounding epidermal tissue. In lin-15 mutant animals, it is commonly observed that two adjacent VPCs both assume the $2^{\circ}$ fate, but it is rarely observed that adjacent VPCs both assume the $1^{\circ}$ fate (Table $|E|$ ). This suggests the action of a signal that prevents adjacent VPCs from both assuming the $1^{\circ}$ fate. The action of this signal can also be observed in experiments in which groups of VPCs are isolated in a lin- 15 mutant animal by laser ablation of the other VPCs. A single isolated VPC in a lin-15 mutant animal assumes the $1^{\circ}$ fate (Table IE3). However, when two adjacent VPCs are left after surgery, one of them will assume the $1^{\circ}$ fate and the other will assume the $2^{\circ}$ fate (Table IE4). The lateral signal acts only at a short range and may require direct cell contact since two isolated VPCs in a lin- 15 mutant are only inhibited from both assuming the $1^{\circ}$ fate if they are close to each other (Table 1E5).

This lateral signal is believed to act through the gene lin12 (Sternberg and Horvitz, 1989). lin- 12 encodes a cell surface protein homologous to the protein encoded by the Notch locus of Drosophila (Greenwald, 1985; Yochem et al., 1988). Both $\mathrm{lin}-12$ and Notch function in lateral interactions that control cell fate choice between cells of equivalent developmental potential (Greenwald et al., 1983;
Greenwald and Rubin, 1992; see P. Simpson, this volume). lin- 12 acts during vulval development to promote the $2^{\circ}$ fate. In animals homozygous for $\mathrm{lin}-12$ loss-of-function mutations, no VPCs assume the $2^{\circ}$ fate, and in animals homozygous for $\mathrm{lin}-12$ gain-of-function mutations, all six VPCs assume the $2^{\circ}$ fate independently of the inductive signal (Table 1F; Greenwald et al., 1983; Sternberg and Horvitz, 1989). lin-12 also promoles the $2^{\circ}$ fate in the AC $\left(1^{\circ}\right.$ fate)-Ventral Uterine (VU) precursor ( $2^{\circ}$ fate) equivalence group (Greenwald et al., 1983). Genetic mosaic analysis of lin- $/ 2$ activity in the AC-VU decision indicates that lin-12 acts in a cell autonomous manner to specify the $2^{\circ}$ fate (Seydoux and Greenwald, 1989). It has thus been predicted that the $\operatorname{lin}-12$ product acts as the receptor for the lateral signal during vulval development.

Lateral signalling acts through a separate pathway from the inductive pathway. It is not clear precisely where the two pathways converge to control VPC fate although it is likely downstream of $\mathrm{lin}-2$, lin-7, and lin-10 (Sternberg and Horvitz, 1989). Currently other components of the lateral pathway, including the ligand for $l i n-12$ and genes that act downstream of $/ \mathrm{in}-\mathrm{I} 2$, are not well characterized. One explanation for this is that these other components of the lateral pathway may be required for an essential decision prior to the time of vulval development. The C. elegans gene glp-I is structurally very similar to lin- $/ 2$ and both genes may have originated from a gene duplication of a common ancestor (Yochem and Greenwald, 1989). $g l p-l$ is required zygotically for maintenance of the mitotic germline (Austin and Kimble, 1987) and is required maternally for the proper fate specification of early blastomeres (Priess et al., 1987). The $1 \mathrm{in}-12 \mathrm{~g} / \mathrm{p}-1$ double mutant has a synthetic zygotic lethal phenotype called Lag (Lambie and Kimble, 1991b). This indicates that $g / p-I$ and $l i n-12$ are functionally redundant for certain essential developmental processes. Other genes that can be mutated to give a Lag phenotype could be shared components of the $l i n-12$ and $g l p-l$ pathways and thus might be required for lateral interactions during vulval development (Lambie and Kimble, 1991b).

\section{PATTERNING OF VULVAL FATES}

Vulval development allows the examination of how different intercellular signals interact to control pattern formation. During vulval development, three intercellular signals direct the six VPCs to adopt the pattern of fates $3^{\circ}$ $3^{\circ} 2^{\circ} 1^{\circ} 2^{\circ} 3^{\circ}$. Mutations that disrupt any of the three signals affects the fates assumed by the VPCs. The wild-type pattern is precise in the number of VPCs induced to assume the $1^{\circ}$ and $2^{\circ}$ vulval fates, the position of the VPCs induced to assume vulval fates, and in the pattern of $1^{\circ}$ and $2^{\circ}$ fates assumed by the induced cells. This section summarizes our knowledge of what signals control the extent, location, and pattern of vulval induction.

\section{Extent}

As mentioned previously, the genetic dose of lin-3 is proposed to control the number of VPCs induced to assume vulval fates. This hypothesis is based on the observations that reduction-of-function mutations in $l i n-3$ reduce the 
number of the VPCs that assume vulval fates and that multicopy lin-3 transgenes, which presumably overexpress the Lin-3 protein, increase the number of VPCs that assume vulval fates (Ferguson and Horvitz, 1985; Hill and Sternberg, 1992). In lin-/2 mutant animals in which lateral signalling is disrupted, the number of VPCs that assume vulval fates is near the wild-type level (Table IF1; Sternberg and Horvitz, 1989). Thus the lateral signal does not appear to regulate the number of VPCs that assume vulval fates. The inhibitory signal acts in a spatially general manner to inhibit vulval fates, but the action of the inhibitory signal is overridden by the inductive signal. Thus the inhibitory signal ensures that vulval fates are assumed only in response to the inductive signal, but does not itself determine the number of VPCs that assume vulval fates.

\section{Location}

The anchor cell controls the location of vulval development. This is illustrated by the pattern of fates assumed by the VPCs in dig- I mutant animals. In dig- I mutants the position of the gonad and the anchor cell can be shifted anteriorly and/or dorsally of their wild-type positions (Thomas et al.. 1990). In animals in which the anchor cell is shifted anteriorly of its wild-type position and remains ventral, the pattern of vulval fates is shifted commensurably. For example. when the anchor cell is positioned over P5.p, P5.p assumes the $1^{\circ}$ fate and the overall pattern of vulval fates is $3^{\circ} 2^{\circ} 1^{\circ}$ $2^{\circ} 3^{\circ} 3^{\circ}$ (Table IG).

\section{Pattern}

As just discussed, in wild-type development, the induction of $1^{\circ}$ and $2^{\circ}$ vulval fates is limited to P5.p, P6.p and P7.p because the anchor cell is located above P6.p and because it only makes enough inductive signal to induce the three most proximal VPCs. What signals direct the three induced VPCs to assume a $2^{\circ} 1^{\circ} 2^{\circ}$ pattern of fates? The experimental analysis of animals that have only one VPC indicates that the inductive signal plays an important role in patterning. The fate of a single, isolated VPC correlates with its distance from the anchor cell: a single VPC that is relatively close to the anchor cell will assume the $1^{\circ}$ fate, a single VPC that is further away will assume the $2^{\circ}$ fate, and a single VPC that is distant will assume the $3^{\circ}$ fate (Table $1 \mathrm{H}$ ) (Sternberg and Horvitz, 1986; P. Sternberg. unpublished results, and M. Herman, and H. R. Horvitz, personal communication). Since these isolated VPCs have no neighboring VPCs, they should not be subject to the effects of the lateral signal. Thus, the fact that the fate of an isolated VPC correlates with its distance from the anchor cell suggests that there is a gradient of inductive signal centered on the anchor cell, and that the VPCs assume different fates in response to different levels of the inductive signal.

The inhibitory signal mediated through the lin-15 pathway does not have an important role in vulval fate patteming. Although all six VPCs assume vulval fates in lin15 mutant animals, the patterning of fates is still normal. In a lin-15 mutant animal, the inductive signal still promotes P6.p to assume the $1^{\circ}$ fate and the lateral signal still functions to prevent P5.p and P7.p from assuming the $1^{\circ}$ fate. Thus if the anchor cell is present in a lin-15 mutant animal, then P5.p. P6.p and P7.p, will assume the same $2^{\circ}$ $1^{\circ} 2^{\circ}$ pattern of fates that they assume in wild-type animals (Table IE1). However, if the anchor cell is ablated, then P5.p. P6.p, and P7.p can each assume either the $1^{\circ}$ or $2^{\circ}$ fate (Table 1E2) (Sternberg, 1988a).

The lateral signal appears to play an important role in the patterning of vulval fates assumed by the induced VPCs. In lin-12 loss-of-function mutant animals, the pattern of fates is usually $3^{\circ} 3^{\circ} 1^{\circ} 1^{\circ} 1^{\circ} 3^{\circ}$ (Table $|\mathrm{F}|$ ). Thus, lateral signalling may be necessary to specify the $2^{\circ}$ fate. Moreover, the lateral signal is believed to measure differences between two VPCs in the amount of inductive signal received and to act in response to this difference to direct the VPCs to assume different fates. This model is supported by indirect evidence that certain levels of the inductive signal can specify a VPC to have different fates depending upon the states of the VPC's neighbors. For example, a VPC in the position of P5.p always assumes the $2^{\circ}$ fate in wild-type development. But an isolated VPC in this position has been observed to assume the $1^{\circ}$ fate in animals in which the other VPCs have been ablated (P. Sternberg, unpublished observations). This isolated VPC may assume the $1^{\circ}$ fate for either of two reasons; it might be receiving more inductive signal than it does in wild type because it lacks neighbors that absorb the inductive signal, or it may receive the same amount of inductive signal as it does in wild type, but lacks lateral signalling from its neighbors that specify it to be $2^{\circ}$. Another example is the pattern of vulval fates in dig- 1 mutant animals in which the anchor cell is displaced to the dorsal side of the animal (Thomas et al., 1990). In these animals the VPC closest to the anchor cell is at a distance equal to or greater than the distance of P5.p is to the anchor cell in a wild-type animal. Thus, no VPC in these dig-I mutant animals should receive more inductive signal then does P5.p in a wild-type animal. Yet, although P5.p always assumes the $2^{\circ}$ fate in wild-type development, VPCs at an equivalent distance from the anchor cell in dig- $l$ mutants often assume the $1^{\circ}$ fate. Thus it is possible that there are intermediate doses of the inductive signal that will specify a VPC to have the $1^{\circ}$ fate in the context of neighboring VPCs that are receiving less inductive signal, but that will specify a VPC to have the $2^{\circ}$ fate in the context of neighboring VPCs that are receiving more inductive signal.

The action of the lateral signal in vulva development is also modeled on the role of lin-12 in the AC-VU equivalence group (Greenwald et al., 1983). In this equivalence group, either the cell Z1.ppp or Z4.aaa can assume the fate of the anchor cell with approximately equal likelihood. Lateral signalling among these two cells mediates this decision without apparent influence from outside cells (Kimble, 1981; Seydoux and Greenwald, 1989). Although either cell can assume either fate, the lateral signal ensures that only one cell always assumes the fate of the anchor cell and that the other cell always assumes the fate of $\mathrm{VU}$ (Kimble and Hirsh, 1979). Based upon this observation, it has been proposed that the lateral signal acts in a feedback loop that establishes mutually exclusive states in adjacent cells. In this model both cells start with the ability to produce both the lateral signal and the receptor for the lateral signal. Cells that receive the lateral signal down-regulate the activity of the lateral signal and up-regulate the activity of the receptor. Conversely, cells that produce the lateral signal 
up-regulate the activity of the lateral signal and downregulate the activity of the receptor. This feedback mechanism should result in only one cell producing active lateral signal and the other cell producing only active receptor (Seydoux and Greenwald, 1989; Sternberg, 1988b). In vulval development, the outcome of lateral signalling among the VPCs is invariant and is believed to be biased by the inductive signal produced by the anchor cell. It is proposed that the lateral signalling initially acts in proportion to the amount of inductive signal received by each VPC. The feedback mechanisms of the lateral signal then act to accentuate the differences in the amount of lateral signalling between the VPCs. This ensures that adjacent cells assume different fates in response to different levels of the inductive signal (Sternberg and Horvitz, 1989).

Another way of comparing the relative contribution of the inductive signal and the lateral signal in controlling pattern formation is by examining the genetic interactions of the two signals. In animals with lin- $/ 2$ gain-of function mutations, all six VPCs usually assume the $2^{\circ}$ fate. There is also usually no anchor cell in these animals and thus no inductive signal (Table 1F2). However, in those lin-12 gain-of-function mutant animals that have an anchor cell, P6.p always assumes the $1^{\circ}$ fate (Table 1F3) (Sternberg and Horvitz, 1989). Thus, for a single cell, the action of the inductive signal in specifying the $1^{\circ}$ fate overrides the action of the lateral signal in specifying the $2^{\circ}$ fate. A function of the lateral signal in patterning though, is to prevent two adjacent VPCs from both assuming the $1^{\circ}$ fate. Thus an important experiment is to determine if the lateral signal can still promote adjacent cells to assume different fates when they both receive high levels of the inductive signal. In lin-3 transgenic animals, which overexpress the inductive signal in the anchor cell, adjacent VPCs often both have the $1^{\circ}$ fate (Table 1I) (Hill and Sternberg, unpublished data). In this situation, two adjacent VPCs both assume the $1^{\circ}$ fate, even though each of them should be laterally signalling their neighbors to be $2^{\circ}$. Thus, either a high level of the inductive signal specifies the $1^{\circ}$ fate and overrides the action of the lateral signal, or, alternatively, Lin-3 protein might bind to, and inhibit the action of either the ligand or the receptor of the lateral signal. In either case, the dose of the inductive signal must be limited to achieve wild-type patterning in which P5.p and P7.p assume the $2^{\circ}$ fate.

\section{Pattern Summary}

The pattern of $1^{\circ}$ and $2^{\circ}$ fates assumed by the VPCs is determined both by the action of an inductive signal encoded by lin-3, and a lateral signal that acts though lin-12. The inductive signal appears to provide the specific information that sets up the pattern of fates assumed by the VPCs. First, the inductive signal originates only from the anchor cell and is the only signal that clearly provides spatially specific information. Second, the fact that the fate of an isolated VPC correlates with its distance from the anchor cell, suggests that different doses of the inductive signal promote the $1^{\circ}$, $2^{\circ}$, and $3^{\circ}$ fates, and that a spatial gradient of the inductive signal promotes the VPCs to assume a graded pattern of fates. Third, a high level of the inductive signal specifies the $1^{\circ}$ fate and overrides the action of the lateral signal in spec- ifying the $2^{\circ}$ fate. This indicates that the production of the inductive signal must be limited to get VPCs with the $2^{\circ}$ fate. The properties of the lateral signal and lin-12 are intriguing. On the one hand the lateral signal seems to reinforce a pattern that has already been determined by the inductive signal; i. e. the lateral signal ensures that P5.p and P7.p assume the $2^{\circ}$ fate in response to intermediate amounts of the inductive signal, and that P6.p assumes the $1^{\circ}$ fate in response to high amounts of the inductive signal. On the other hand, wild-type activity of the gene lin-12, which is believed to encode the receptor for the lateral signal, is required to specify the $2^{\circ}$ fate. Thus a VPC that receives a dose of the inductive signal that promotes the $2^{\circ}$ fate still apparently requires input from the lateral signal to assume the $2^{\circ}$ fate. Thus, the mechanism by which an isolated VPC assumes the $2^{\circ}$ fate is unclear, since it lacks neighbors with which it can laterally signal. Such a VPC may engage in autocrine signalling through lin- 12 to assume the $2^{\circ}$ fate. The inductive signal and the lateral signal appear to cooperate to promote the graded $2^{\circ} 1^{\circ} 2^{\circ}$ pattern of fates assumed by the induced VPCs. The use of two signals to promote this pattern may ensure that the process of pattern formation has a reproducible outcome. Thus for example, the lateral signal could ensure that the VPCs assume a $2^{\circ} 1^{\circ}$ $2^{\circ}$ pattern of fates over a broad dose range of the inductive signal, as long as the inductive signal is distributed in a gradient.

Our current model of VPC fate determination is summarized in Fig. 1. An inhibitory signal that arises in a spatially general manner inhibits the VPCs from assuming vulval fates in the absence of the inductive signal. Localized production of the inductive signal encoded by lin-3 controls the number and location of VPCs that assume the vulval fates. Lateral signalling among the VPCs acts in response to a spatial gradient of inductive signal to specify P5.p and P7.p to assume the $2^{\circ}$ fate.

\section{PROSPECTS}

A question for the future is how the different signals interact to control the fate of the VPCs. The molecular cloning of several key genes in these intercellular pathways means that the interactions of these signals can be analyzed both genetically and by the techniques of molecular biology. The molecular mechanism by which the inhibitory signal represses the response pathway of the VPCs has not been established. Vulva development provides an example where the outcome of lateral interactions among cells of equivalent developmental potential appears to be controlled by the action of an outside signal. Elucidating the mechanisms by which the lateral signal and inductive signal interact to control the choice between $1^{\circ}$ and $2^{\circ}$ will be a goal of future research. The use of genetics to identify additional components of the lateral signalling pathway will be important in understanding the mechanism of action of the lateral signal. Genetic screens for suppressers of existing mutations that disrupt vulval development should be useful in identifying new genes involved in the pathways of vulval fate determination. 


\section{REFERENCES}

Aroian, R. V., Koga, M., Mendel, J. E., Ohshima, Y. and Sternberg, P. W. (1990). The let-23 gene necessary for Caenorhabditis elegans vulval induction encodes a tyrosine kinase of the EGF receptor subfamily. Nature 348, 693-699.

Aroian, R. V. and Sternberg, P. W. (1991). Multiple functions of let-23, a C. elegans receptor tyrosine kinase gene required for vulval induction. Genetics 128, 251-267.

Austin, J. and Kimble, J. (1987). glp-1 is required in the germ line for regulation of the decision between mitosis and meiosis in C. elegans. Cell 51. 589-599.

Beitel, G., Clark, S. and Horvitz, H. R. (1990). The Caenorhabditis elegans ras gene let-60 acts as a switch in the pathway of vulval induction. Nature 348, 503-509.

Brenner, S. (1974). The genetics of Caenorhabditis elegans. Genetics 77. $71-94$.

Carpenter, G. and Wahl, M. I. (1990). The epidermal growth factor family. In Peptide Growth Factors and Their Receptors. Vol. 95 of Handbook of Experimental Pharmacology (eds Sporn, M. B. and Roberts. A. B.). pp. 69-171. Heidelberg: Springer-Verlag.

Clark, D. V., Rogalski, T. M., Donati, L. M. and Baillie, D. L. (1988). The unc-22 (IV) region of Caenorhabditis elegans: genetic analysis of lethal mutations. Genetics 119, 345-353.

Clark, S. G., Stern, M. J. and Horvitz, H. R. (1992). C. elegans cellsignalling gene sem-5 encodes a protein with $\mathrm{SH} 2$ and $\mathrm{SH} 3$ domains. Nature 356, 340-344.

Davis, C. G. (1990). The many faces of epidermal growth factor repeats. New Biol. 2, 410-419.

Derynck, R., Roberts, A. B., Winkler, M. E., Chen, E. Y. and Goeddel, D. V. (1984). Human transforming growth factor-alpha: precursor structure and expression in E. coli. Cell 38, 287-297.

Ferguson, E. and Horvitz, H. R. (1985). Identification and characterization of 22 genes that affect the vulval cell lineages of Caenorhabditis elegans. Genetics 110, 17-72.

Ferguson, E. and Horvitz, H. R. (1989). The multivulva phenotype of certain C. elegans mutants results from defects in two functionallyredundant pathways. Genetics 123, 109-121.

Ferguson, E. L., Sternberg, P. W. and Horvitz, H. R. (1987). A genetic pathway for the specification of the vulval cell lineages of Caenorhabditis elegans. Nature 326, 259-267.

Greenwald, I. and Rubin, G. M. (1992). Making a difference: the role of cell-cell interactions in establishing separate identities for equivalent cells. Cell 68, 271-281.

Greenwald, I. S. (1985). lin-12, a nematode homeotic gene, is homologous to a set of mammalian proteins that includes epidermal growth factor. Cell $43,583-590$.

Greenwald, I. S., Sternberg, P. W. and Horvitz, H. R. (1983). The lin-12 locus specifies cell fates in Caenorhabditis elegans. Cell 34, 435-444.

Gregory, H. (1975). Isolation and structure of urogastrone and its relationship to epidermal growth factor. Nature 257, 325-327.

Han, M., Aroian, R. and Sternberg, P. W. (1990). The let-60 locus controls the switch between vulval and non-vulval cell types in $C$. elegans. Genetics 126, 899-913.

Han, M., Golden, A., Han, Y. and Sternberg, P. W. (1993). C. elegans lin45 raf gene participates in let-60 ras stimulated vulval differentiation. Nature 363, 133-140.

Han, M. and Sternberg, P. W. (1990). let-60, a gene that specifies cell fates during C. elegans vulval induction, encodes a ras protein. Cell 63, 921 931.

Herman, R. K. (1978). Crossover suppressors and balanced recessive lethals in Caenorhabditis elegans. Genetics 88, 49-65.

Herman, R. K. and Hedgecock, E. M. (1990). The size of the C. elegans vulval primordium is limited by lin-15 expression in surrounding hypodermis. Nature 348, 169-171.

Higashiyama, S., Abraham, J. A., Miller, J., Fiddes, J. C. and Klagsbrun, M. (1991). A heparin-binding growth factor secreted by macrophage-like cells that is related to EGF. Science 251. 936-939.

Hill, R. J. and Sternberg, P. W. (1992). The lin-3 gene encodes an inductive signal for vulval development in C. elegans. Nature 358, 470476.

Holmes, W. E., Sliwkowski, M. X., Akita, R. W., Henzel, W. J., Lee, J., Park, J. W., Yansura, D., Abadi, N., Raab, H., Lewis, G. D., Shepard, H. M., Kuang, W., Wood, W. I., Goeddel, D. V. and Vandlen, R. L.
(1992). Identification of heregulin, a specific activator of p185 erbB2. Science. 256, 1205-1210.

Horvitz, H. R. and Sulston, J. E. (1980). Isolation and genetic characterization of cell-lineage mutants of the nematode Caenorhabditis elegans. Genetics 96. 435-454.

Horvitz, H. R. (1988), Genetics of cell lineage. In The Nematode Caenorhabditis elegans (ed. W. B. Wood), pp. 157-190. Cold Spring Harbor. New York: Cold Spring Harbor Laboratory.

Horvitz, H. R. and Sternberg, P. W. (1991). Multiple intercellular signalling systems control the development of the C. elegans vulva. Nature 351, 535-541.

Kim, S. K. and Horvitz, H. R. (1990). The Caenorhabditis elegans gene lin-10 is broadly expressed while required specifically for the determination of vulval cell fates. Genes Dev. 4, 357-371.

Kimble, J. (1981). Lineage alterations after ablation of cells in the somatic gonad of Caenorhabditis elegans. Dev. Biol. 87, 286-300.

Kimble, J. and Hirsh, D. (1979). Post-embryonic cell lineages of the hermaphrodite and male gonads in Caenorhabditis elegans. Dev. Biol. 70 , 396-417.

Lambie, E. J. and Kimble, J. (1991a). Genetic control of cell interactions in nematode development. Ann. Rev. Genet. 25, 411-436.

Lambie, E. J. and Kimble, J. (199 Ib). Two homologous regulatory genes, lin- 12 and $g l p-1$, have overlapping functions. Development 112, 231-240.

Massagué, J. (1990). Transforming growth factor- $\alpha$. J. Biol. Chem. 265, 21393-21396.

McCormick, F. (1993). How receptors turn Ras on. Nature 363, 15-16.

Mello, C. C., Kramer, J. M., Stinchcomb, D. and Ambros, V. (1991). Efficient gene transfer in C. elegans after microinjection of DNA into germline cytoplasm: extrachromosomal maintenance and integration of transforming sequences, EMBO J. 10, 3959-3970.

Priess, J., Schnabel, H. and Schnabel, R. (1987). The glp-/ locus and cellular interactions in early Caenorhabditis elegans embryos. Cell $\mathbf{5 1}$, 601-611

Priess, J. R. and Thomson, J. N. (1987). Cellular interactions in early Caenorhabditis elegans embryos. Cell 48, 241-250.

Rutledge, B. J., Zhang, K., Bier, E., Jan, Y. N. and Perrimon, N. (1992). The Drosophila spitz gene encodes a putative EGF-like growth factor involved in dorsal-ventral axis formation and neurogenesis. Genes Dev. 6 , 1503-1517.

Savage, C. R. J., Inagami, T. and Cohen, S. (1972). The primary structure of epidermal growth factor. J. Biol. Chem. 247. 7612-7621.

Schnabel, R. (1991). Cellular interactions involved in the determination of the early C. elegans embryo. Mech. Dev. 34, 85-100.

Seydoux, G. and Greenwald, I. (1989). Cell autonomy of lin- 12 function in a cell fate decision in C. elegans. Cell 57, 1237-1245.

Shoyab, M., Plowman, G. D., McDonald, V. L., Bradley, J. G. and Todaro, G. J. (1989). Structure and function of human amphiregulin: a member of the epidermal growth factor family. Science 243, 1074-1076.

Sternberg, P. W. (1988a). Lateral inhibition during vulval induction in Caenorhabditis elegans. Nature 335, 551-554.

Sternberg, P. W. (1988b). Control of cell fates in equivalence groups in $C$. elegans. Trends Neurosci. 11, 259-264.

Sternberg, P. W. and Horvitz, H. R. (1986). Pattern formation during vulval development in Caenorhabditis elegans. Cell 44, 761-772.

Sternberg, P. W. and Horvitz, H. R. (1989). The combined action of two intercellular signalling pathways specifies three cell fates during vulval induction in C. elegans. Cell 58, 679-693.

Stringham, E. G., Dixon, D. K., Jones, D. and Candido, E. P. M. (1992). Temporal and spatial expression patterns of the small heat shock (hspl6) genes in transgenic Caenorhabditis elegans. Mol. Biol. Cell 3. 221-233.

Sulston, J. E. (1976). Post-embryonic development in the ventral cord of Caenorhabditis elegans. Philos. Trans. R. Soc. Lond. B 275, 287-298.

Sulston, J. E., Albertson, D. G. and Thomson, J. N. (1980). The Caenorhabditis elegans male: Postembryonic development of nongonadal structures. Dev. Biol. 78, 542-576.

Sulston, J. and Horvitz, H. R. (1977). Postembryonic cell lineages of the nematode Caenorhabditis elegans. Dev. Biol. 56, 110-156.

Sulston, J. E. and Horvitz, H. R. (1981). Abnormal cell lineages in mutants of the nematode Caenorhabditis elegans. Dev. Biol. 82, 41-55.

Sulston, J. E., Schierenberg, E., White, J. G. and Thomson, J. N. (1983) The embryonic cell lineage of the nematode Caenorhabditis elegans. Dev. Biol. 100, 64-119.

Sulston, J. E. and White, J. G. (1980). Regulation and cell autonomy 
during postembryonic development of Caenorhabditis elegans. Dev. Biol. 78, 577-597.

Thomas, J. H., Stern, M. J. and Horvitz, H. R. (1990). Cell interactions coordinate the development of the C. elegans egg-laying system. Cell 62, 1041-1052.

Wen, D., Peles, E., Cupples, R., Suggs, S. V., Bacus, S. S., Luo, Y., Trail, G., Hu, S., Silbiger, S. M., Levy, R. B., Koski, R. A., Lu, H. S. and Yarden, Y. (1992). Neu differentiation factor: a transmembrane glycoprotein containing an EGF domain and an immunoglobulin homology unit. Cell 69, 559-572.

Yochem, J. and Greenwald, I. (1989). g/p- $/$ and $l i n-12$, genes implicated in distinct cell-cell interactions in $C$. elegans, encode similar transmembrane proteins. Cell 58, 553-563.

Yochem, J., Weston, K. and Greenwald, I. (1988). C. elegans lin-12 encodes a transmembrane protein similar to Drosophila Notch and yeast cell cycle gene products. Nature $335,547-550$. 